\title{
INTEGRAL GROUP RING OF THE FIRST MATHIEU SIMPLE GROUP
}

\author{
VICTOR BOVDI* and ALEXANDER KONOVALOV ${ }^{\dagger 1}$ \\ *Institute of Mathematics, University of Debrecen, P.O. Box 12, H-4010 Debrecen; \\ Institute of Mathematics and Informatics, College of Nyíregyháza, Sóstói út 31/b, H-4410 \\ Nyíregyháza, Hungary \\ $\dagger$ Department of Mathematics, Zaporozhye National University, 66 Zhukovskogo str., 69063, \\ Zaporozhye, Ukraine; current address: Department of Mathematics, Vrije Universiteit \\ Brussel, Pleinlaan 2, B-1050 Brussel, Belgium \\ Email: alexander.konovalov@gmail.com
}

\begin{abstract}
We investigate the classical Zassenhaus conjecture for the normalized unit group of the integral group ring of the simple Mathieu group $M_{11}$. As a consequence, for this group we confirm the conjecture by Kimmerle about prime graphs.
\end{abstract}

\section{Introduction and main results}

Let $V(\mathbb{Z} G)$ be the normalized unit group of the integral group ring $\mathbb{Z} G$ of a finite group $G$. The following famous conjecture was formulated by H. Zassenhaus in [15]:

Conjecture 1 (ZC) Every torsion unit $u \in V(\mathbb{Z} G)$ is conjugate within the rational group algebra $\mathbb{Q} G$ to an element of $G$.

This conjecture is already confirmed for several classes of groups but, in general, the problem remains open, and a counterexample is not known.

Various methods have been developed to deal with this conjecture. One of the original ones was suggested by I. S. Luthar and I. B. S. Passi [12, 13], and it was improved further by M. Hertweck [9]. Using this method, the conjecture was proved for several new classes of groups, in particular for $S_{5}$ and for some finite simple groups (see $[4,9,10,12,13]$ ).

The Zassenhaus conjecture appeared to be very hard, and several weakened variations of it were formulated (see, for example, [3]). One of the most interesting modifications was suggested by W. Kimmerle [11]. Let us briefly introduce it now.

Let $G$ be a finite group. Denote by \# $(G)$ the set of all primes dividing the order of $G$. Then the Gruenberg-Kegel graph (or the prime graph) of $G$ is a graph $\pi(G)$ with vertices labelled by primes from \# $(G)$, such that vertices $p$ and $q$ are adjacent if and only if there is an element of order $p q$ in the group $G$. Then the conjecture by Kimmerle can be formulated in the following way:

Conjecture 2 (KC) If $G$ is a finite group, then $\pi(G)=\pi(V(\mathbb{Z} G))$.

\footnotetext{
${ }^{1}$ The research was supported by OTKA T 037202, T 038059 and ADSI107(VUB).
} 
For Frobenius groups and solvable groups this conjecture was confirmed in [11]. In the present paper we continue the investigation of $(\mathbf{K C})$, and confirm it for the first simple Mathieu group $M_{11}$, using the Luthar-Passi method. Moreover, this allows us to give a partial solution of $(\mathbf{Z C})$ for $M_{11}$.

Our main results are the following:

Theorem 1 Let $V(\mathbb{Z} G)$ be the normalized unit group of the integral group ring $\mathbb{Z} G$, where $G$ is the simple Mathieu group $M_{11}$. Let $u$ be a torsion unit of $V(\mathbb{Z} G)$ of order $|u|$. We have:

(i) if $|u| \neq 12$, then $|u|$ coincides with the order of some element $g \in G$;

(ii) if $|u| \in\{2,3,5,11\}$, then $u$ is rationally conjugate to some $g \in G$;

(iii) if $|u|=4$, then the tuple of partial augmentations of $u$ belongs to the set

$$
\begin{aligned}
& \left\{\left(\nu_{2 a}, \nu_{3 a}, \nu_{4 a}, \nu_{6 a}, \nu_{5 a}, \nu_{8 a}, \nu_{8 b}, \nu_{11 a}, \nu_{11 b}\right) \in \mathbb{Z}^{9} \mid \nu_{k x}=0,\right. \\
& \left.k x \notin\{2 a, 4 a\},\left(\nu_{2 a}, \nu_{4 a}\right) \in\{(0,1),(2,-1)\}\right\} ;
\end{aligned}
$$

(iv) if $|u|=6$, then the tuple of partial augmentations of $u$ belongs to the set

$$
\begin{aligned}
&\left\{\left(\nu_{2 a}, \nu_{3 a}, \nu_{4 a}, \nu_{6 a}, \nu_{5 a}, \nu_{8 a}, \nu_{8 b}, \nu_{11 a}, \nu_{11 b}\right)\right. \in \mathbb{Z}^{9} \mid \nu_{k x}=0, \\
& k x \notin\{2 a, 3 a, 6 a\},\left(\nu_{2 a}, \nu_{3 a}, \nu_{6 a}\right) \in\{(-2,3,0),(0,0,1), \\
&(0,3,-2),(2,-3,2),(2,0,-1)\}\} ;
\end{aligned}
$$

(v) if $|u|=8$, then the tuple of partial augmentations of $u$ belongs to the set

$$
\begin{aligned}
\left\{\left(\nu_{2 a}, \nu_{3 a}, \nu_{4 a}, \nu_{6 a}, \nu_{5 a}, \nu_{8 a}, \nu_{8 b}, \nu_{11 a}, \nu_{11 b}\right) \in \mathbb{Z}^{9} \mid \nu_{k x}=0\right. \\
k x \notin\{4 a, 8 a, 8 b\}, \quad\left(\nu_{4 a}, \nu_{8 a}, \nu_{8 b}\right) \in\{(0,0,1),(0,1,0), \\
(2,-1,0),(2,0,-1)\}\} ;
\end{aligned}
$$

(vi) if $|u|=12$, then the tuple of partial augmentations of $u$ cannot belong to the set

$$
\begin{aligned}
\mathbb{Z}^{9} \backslash\left\{\left(\nu_{2 a}, \nu_{3 a}, \nu_{4 a}, \nu_{6 a}, \nu_{5 a}, \nu_{8 a}, \nu_{8 b}, \nu_{11 a}, \nu_{11 b}\right)\right. & \in \mathbb{Z}^{9} \mid \nu_{k x}=0, \\
k x \notin\{2 a, 4 a, 6 a\},\left(\nu_{2 a}, \nu_{4 a}, \nu_{6 a}\right) & \in\{(-1,1,1),(1,1,-1)\}\} .
\end{aligned}
$$

Corollary 1 Let $V(\mathbb{Z} G)$ be the normalized unit group of the integral group ring $\mathbb{Z} G$, where $G$ is the simple Mathieu group $M_{11}$. Then $\pi(G)=\pi(V(\mathbb{Z} G))$, where $\pi(G)$ and $\pi(V(\mathbb{Z} G))$ are prime graphs of $G$ and $V(\mathbb{Z} G)$, respectively. Thus, for $M_{11}$ the conjecture by Kimmerle is true.

\section{Notation and preliminaries}

Let $u=\sum \alpha_{g} g$ be a normalized torsion unit of order $k$ and let $\nu_{i}=\varepsilon_{C_{i}}(u)$ be a partial augmentation of $u$. By S. D. Berman's Theorem [2] we have that $\nu_{1}=0$ and

$$
\nu_{2}+\nu_{3}+\cdots+\nu_{m}=1
$$


For any character $\chi$ of $G$ of degree $n$, we have that $\chi(u)=\sum_{i=2}^{m} \nu_{i} \chi\left(h_{i}\right)$, where $h_{i}$ is a representative of the conjugacy class $C_{i}$.

We need the following results:

Proposition 1 (see [12]) Suppose that $u$ is an element of $\mathbb{Z} G$ of order $k$. Let $z$ be a primitive $k$-th root of unity. Then for every integer $l$ and any character $\chi$ of $G$, the number

$$
\mu_{l}(u, \chi)=\frac{1}{k} \sum_{d \mid k} \operatorname{Tr}_{\mathbb{Q}\left(z^{d}\right) / \mathbb{Q}}\left\{\chi\left(u^{d}\right) z^{-d l}\right\}
$$

is a non-negative integer.

Proposition 2 (see [6]) Let $u$ be a torsion unit in $V(\mathbb{Z} G)$. Then the order of $u$ divides the exponent of $G$.

Proposition 3 (see [12] and Theorem 2.7 in [14]) Let $u$ be a torsion unit of $V(\mathbb{Z} G)$. Let $C$ be a conjugacy class of $G$. If $p$ is a prime dividing the order of a representative of $C$ but not the order of $u$ then the partial augmentation $\varepsilon_{C}(u)=0$.

M. Hertweck (see [10], Proposition 3.1; [9], Lemma 5.6) obtained the next result:

Proposition 4 Let $G$ be a finite group and let $u$ be a torsion unit in $V(\mathbb{Z} G)$.

(i) If $u$ has order $p^{n}$, then $\varepsilon_{x}(u)=0$ for every $x$ of $G$ whose p-part is of order strictly greater than $p^{n}$.

(ii) If $x$ is an element of $G$ whose p-part, for some prime $p$, has order strictly greater than the order of the p-part of $u$, then $\varepsilon_{x}(u)=0$.

Note that the first part of Proposition 4 gives a partial answer to the conjecture by A. Bovdi (see [1]). Also M. Hertweck ([9], Lemma 5.5) gives a complete answer to the same conjecture in the case when $G=\operatorname{PSL}(2, \mathbb{F})$, where $\mathbb{F}=\operatorname{GF}\left(p^{k}\right)$.

In the rest of the paper, for the partial augmentation $\nu_{i}$ we shall also use the notation $\nu_{k x}$, where $k$ is the order of the representative of the $i$-th conjugacy class, and $x$ is a distinguishing letter for this particular class with elements of order $k$.

Proposition 5 (see [12] and Theorem 2.5 in [14]) Let $u$ be a torsion unit of $V(\mathbb{Z} G)$ of order $k$. Then $u$ is conjugate in $\mathbb{Q} G$ to an element $g \in G$ if and only if for each $d$ dividing $k$ there is precisely one conjugacy class $C_{i_{d}}$ with partial augmentation $\varepsilon_{C_{i_{d}}}\left(u^{d}\right) \neq 0$.

Proposition 6 (see [6]) Let $p$ be a prime, and let $u$ be a torsion unit of $V(\mathbb{Z} G)$ of order $p^{n}$. Then for $m \neq n$ the sum of all partial augmentations of $u$ with respect to conjugacy classes of elements of order $p^{m}$ is divisible by $p$.

The Brauer character table modulo $p$ of the group $M_{11}$ will be denoted by $\mathfrak{B C T}(p)$. 


\section{Proof of the Theorem}

It is well known $[7,8]$ that $|G|=2^{4} \cdot 3^{2} \cdot 5 \cdot 11$ and $\exp (G)=1320$. The character table of $G$, as well as the Brauer character tables $\mathfrak{B C T}(p)$, where $p \in\{2,3,5,11\}$, can be found using the computational algebra system GAP [7].

Since the group $G$ possesses elements of orders 2, 3, 4, 5, 6, 8 and 11, first of all we shall investigate units with these orders. After this, by Proposition 2, the order of each torsion unit divides the exponent of $G$, so it will be enough to consider units of orders $10,12,15,22,24,33$ and 55 , because if $u$ will be a unit of another possible order, then there is $t \in \mathbb{N}$ such that $u^{t}$ has an order from this list. We shall prove that units of all these orders except 12 do not appear in $V(\mathbb{Z} G)$. For units of order 12 we are not able to prove this, but we reduce this question to only two cases.

Let $u \in V(\mathbb{Z} G)$ have order $k$. By S. D. Berman's Theorem [2] and Proposition 3 we have $\nu_{1 a}=0$ and

$$
\begin{array}{ccl}
\nu_{3 a}=\nu_{5 a}=\nu_{6 a}=\nu_{11 a}=\nu_{11 b}=0 & \text { when } & k=2,4,8 ; \\
\nu_{2 a}=\nu_{4 a}=\nu_{5 a}=\nu_{6 a}=\nu_{8 a}=\nu_{8 b}=\nu_{11 a}=\nu_{11 b}=0 & \text { when } & k=3 ; \\
\nu_{2 a}=\nu_{3 a}=\nu_{4 a}=\nu_{6 a}=\nu_{8 a}=\nu_{8 b}=\nu_{11 a}=\nu_{11 b}=0 & \text { when } & k=5 ; \\
\nu_{5 a}=\nu_{11 a}=\nu_{11 b}=0 & \text { when } & k=6 ; \\
\nu_{2 a}=\nu_{3 a}=\nu_{4 a}=\nu_{5 a}=\nu_{6 a}=\nu_{8 a}=\nu_{8 b}=0 & \text { when } & k=11 ; \\
\nu_{3 a}=\nu_{6 a}=\nu_{11 a}=\nu_{11 b}=0 & \text { when } & k=10 ; \\
\nu_{5 a}=\nu_{11 a}=\nu_{11 b}=0 & \text { when } & k=12 ; \\
\nu_{2 a}=\nu_{4 a}=\nu_{6 a}=\nu_{8 a}=\nu_{8 b}=\nu_{11 a}=\nu_{11 b}=0 & \text { when } & k=15 ; \\
\nu_{2 a}=\nu_{3 a}=\nu_{4 a}=\nu_{5 a}=\nu_{6 a}=0 & \text { when } & k=22 ; \\
\nu_{5 a}=\nu_{11 a}=\nu_{11 b}=0 & \text { when } & k=24 ; \\
\nu_{2 a}=\nu_{4 a}=\nu_{5 a}=\nu_{6 a}=\nu_{8 a}=\nu_{8 b}=0 & \text { when } & k=33 ; \\
\nu_{2 a}=\nu_{3 a}=\nu_{4 a}=\nu_{6 a}=\nu_{8 a}=\nu_{8 b}=0 & \text { when } & k=55 .
\end{array}
$$

It follows immediately by Proposition 5 that the units of orders 3 and 5 are rationally conjugate to some element of $G$.

Now we consider each case separately:

- Let $u$ be an involution. Then using (3.1) and Proposition 4 we obtain that $\nu_{4 a}=\nu_{8 a}=\nu_{8 b}=0$, so $\nu_{2 a}=1$.

- Let $u$ be a unit of order 4 . Then by (3.1) and Proposition 4 we have $\nu_{2 a}+\nu_{4 a}=1$. By $(2.2), \quad \mu_{0}\left(u, \chi_{3}\right)=\frac{1}{4}\left(-4 \nu_{2 a}+8\right) \geq 0$ and $\mu_{2}\left(u, \chi_{3}\right)=$ $\frac{1}{4}\left(4 \nu_{2 a}+8\right) \geq 0$, so $\nu_{2 a} \in\{-2,-1,0,1,2\}$. Now using the inequalities

$$
\begin{aligned}
& \mu_{0}\left(u, \chi_{5}\right)=\frac{1}{4}\left(6 \nu_{2 a}-2 \nu_{4 a}+14\right) \geq 0 ; \\
& \mu_{2}\left(u, \chi_{5}\right)=\frac{1}{4}\left(-6 \nu_{2 a}+2 \nu_{4 a}+14\right) \geq 0,
\end{aligned}
$$

we get that there are only two integral solutions $\left(\nu_{2 a}, \nu_{4 a}\right) \in\{(0,1),(2,-1)\}$ satisfying (2.1) and Proposition 6, such that all $\mu_{i}\left(u, \chi_{j}\right)$ are non-negative integers.

- Let $u$ be a unit of order 6 . Then by (2.1), (2.2) and Proposition 4 we obtain

$$
\nu_{2 a}+\nu_{3 a}+\nu_{6 a}=1 .
$$


Now, using $\mathfrak{B C T}(11)$ from the system of inequalities $\mu_{0}\left(u, \chi_{6}\right)=\frac{1}{6}\left(-4 \nu_{3 a}+\right.$ $12) \geq 0$ and $\mu_{3}\left(u, \chi_{6}\right)=\frac{1}{6}\left(4 \nu_{3 a}+12\right) \geq 0$, we have that $\nu_{3 a} \in\{-3,0,3\}$. Furthermore, from the system of inequalities

$$
\begin{aligned}
& \mu_{3}\left(u, \chi_{2}\right)=\frac{1}{6}\left(-2 \nu_{2 a}+4 \nu_{6 a}+8\right) \geq 0 \\
& \mu_{0}\left(u, \chi_{2}\right)=\frac{1}{6}\left(2 \nu_{2 a}-4 \nu_{6 a}+10\right) \geq 0 \\
& \mu_{1}\left(u, \chi_{2}\right)=\frac{1}{6}\left(\nu_{2 a}-2 \nu_{6 a}+8\right) \geq 0
\end{aligned}
$$

we get that $\nu_{2 a}-2 \nu_{6 a} \in\{-2,4\}$, so $\nu_{6 a} \in\{-2,-1,0,1,2\}$. Using the inequalities

$$
\begin{aligned}
& \mu_{0}\left(u, \chi_{3}\right)=\frac{1}{6}\left(-4 \nu_{2 a}+2 \nu_{3 a}+2 \nu_{6 a}+10\right) \geq 0 \\
& \mu_{2}\left(u, \chi_{3}\right)=\frac{1}{6}\left(2 \nu_{2 a}-\nu_{3 a}-\nu_{6 a}+7\right) \geq 0,
\end{aligned}
$$

we obtain only the following integral solutions $\left(\nu_{2 a}, \nu_{3 a}, \nu_{6 a}\right)$ :

$$
\{(-2,3,0),(0,0,1),(0,3,-2),(2,-3,2),(2,0,-1)\},
$$

such that all $\mu_{i}\left(u, \chi_{j}\right)$ are non-negative integers.

Using the GAP package LAGUNA [5], we tested all possible $\mu_{i}\left(u, \chi_{j}\right)$ for all tuples $\left(\nu_{2 a}, \nu_{3 a}, \nu_{6 a}\right)$ from (3.2), and were not able to produce a contradiction. Thus, in this case, as well as in the case of elements of order 4, the LutharPassi method is not enough to prove the rational conjugacy.

- Let $u$ be a unit of order 8. By (3.1) and Proposition 4 we have

$$
\nu_{2 a}+\nu_{4 a}+\nu_{8 a}+\nu_{8 b}=1 .
$$

Since $\left|u^{2}\right|=4$, by $(3.2)$ it yields that $\chi_{j}\left(u^{2}\right)=\bar{\nu}_{2 a} \chi_{j}(2 a)+\bar{\nu}_{4 a} \chi_{j}(4 a)$. Now using $\mathfrak{B C T}(3)$, by $(2.2)$ in the case when $\left(\bar{\nu}_{2 a}, \bar{\nu}_{4 a}\right)=(0,1)$ we obtain

$$
\begin{array}{ll}
\mu_{0}\left(u, \chi_{5}\right)=\frac{1}{8}\left(-8 \nu_{2 a}+8\right) \geq 0 ; & \mu_{4}\left(u, \chi_{5}\right)=\frac{1}{8}\left(8 \nu_{2 a}+8\right) \geq 0 ; \\
\mu_{0}\left(u, \chi_{4}\right)=\frac{1}{8}\left(8 \nu_{2 a}+8 \nu_{4 a}+16\right) \geq 0 ; & \mu_{4}\left(u, \chi_{4}\right)=\frac{1}{8}\left(-8 \nu_{2 a}-8 \nu_{4 a}+16\right) \geq 0 ; \\
\mu_{1}\left(u, \chi_{2}\right)=\frac{1}{8}\left(4 \nu_{8 a}-4 \nu_{8 b}+4\right) \geq 0 ; & \mu_{4}\left(u, \chi_{7}\right)=\frac{1}{8}\left(-8 \nu_{8 a}-8 \nu_{8 b}+24\right) \geq 0 ; \\
\mu_{5}\left(u, \chi_{2}\right)=\frac{1}{8}\left(-4 \nu_{8 a}+4 \nu_{8 b}+4\right) \geq 0 ; & \mu_{0}\left(u, \chi_{7}\right)=\frac{1}{8}\left(8 \nu_{8 a}+8 \nu_{8 b}+24\right) \geq 0 .
\end{array}
$$

It follows that $-1 \leq \nu_{2 a} \leq 1,-3 \leq \nu_{4 a} \leq 3, \quad-2 \leq \nu_{8 a}, \nu_{8 b} \leq 2$. Considering the additional inequality

$$
\mu_{0}\left(u, \chi_{2}\right)=\frac{1}{8}\left(4 \nu_{2 a}-4 \nu_{4 a}-4 \nu_{8 a}-4 \nu_{8 b}+4\right) \geq 0,
$$

and using Proposition 6, it is easy to check that this system has the following integral solutions $\left(\nu_{2 a}, \nu_{4 a}, \nu_{8 a}, \nu_{8 b}\right)$ :

$$
\left\{\begin{array}{ccc}
(0,2,0,-1), & (0,2,-1,0), & (0,-2,1,2), \\
(0,-2,2,1), & (0,0,1,0), \quad(0,0,0,1)
\end{array}\right\} .
$$


In the case when $\left(\bar{\nu}_{2 a}, \bar{\nu}_{4 a}\right)=(2,-1)$ using $\mathfrak{B C T}(3)$, by $(2.2)$ we obtained that $\mu_{0}\left(u, \chi_{5}\right)=-\mu_{4}\left(u, \chi_{5}\right)=-\nu_{2 a}=0$ and

$$
\begin{array}{ll}
\mu_{0}\left(u, \chi_{4}\right)=\frac{1}{8}\left(8 \nu_{4 a}+16\right) \geq 0 ; & \mu_{4}\left(u, \chi_{4}\right)=\frac{1}{8}\left(-8 \nu_{4 a}+16\right) \geq 0 ; \\
\mu_{1}\left(u, \chi_{2}\right)=\frac{1}{8}\left(4 \nu_{8 a}-4 \nu_{8 b}+4\right) \geq 0 ; & \mu_{5}\left(u, \chi_{2}\right)=\frac{1}{8}\left(-4 \nu_{8 a}+4 \nu_{8 b}+4\right) \geq 0 .
\end{array}
$$

It is easy to check that $-2 \leq \nu_{4 a}, \nu_{8 a}, \nu_{8 b} \leq 2$, and this system has the following integral solutions:

$$
\left\{\begin{array}{ccc}
(0,2,-1,0), & (0,2,0,-1), & (0,0,0,1), \\
(0,-2,2,1), & (0,0,1,0), & (0,-2,1,2)
\end{array}\right\} .
$$

Now using $\mathfrak{B C T}(11)$ in the case when $\left(\bar{\nu}_{2 a}, \bar{\nu}_{4 a}\right)=(0,1)$, by $(2.2)$ we get

$$
\begin{array}{ll}
\mu_{0}\left(u, \chi_{3}\right)=\frac{1}{8}\left(-8 \nu_{2 a}+8\right) \geq 0 ; & \mu_{4}\left(u, \chi_{3}\right)=\frac{1}{8}\left(8 \nu_{2 a}+8\right) \geq 0 ; \\
\mu_{1}\left(u, \chi_{3}\right)=\frac{1}{8}(4 t+12) \geq 0 ; & \mu_{5}\left(u, \chi_{3}\right)=\frac{1}{8}(-4 t+12) \geq 0 ; \\
\mu_{0}\left(u, \chi_{2}\right)=\frac{1}{8}(4 v-4 w+12) \geq 0 ; & \mu_{4}\left(u, \chi_{2}\right)=\frac{1}{8}(-4 v+4 w+12) \geq 0 ; \\
\mu_{0}\left(u, \chi_{5}\right)=\frac{1}{8}(4 z-4 w+12) \geq 0 ; & \mu_{4}\left(u, \chi_{5}\right)=\frac{1}{8}(-4 z+4 w+12) \geq 0,
\end{array}
$$

where $t=\nu_{8 a}-\nu_{8 b}, z=3 \nu_{2 a}-\nu_{4 a}, v=\nu_{2 a}+\nu_{4 a}$ and $w=\nu_{8 a}+\nu_{8 b}$. From this it follows that $-1 \leq \nu_{2 a} \leq 1,-8 \leq \nu_{2 a} \leq 10,-4 \leq \nu_{8 a}, \nu_{8 b} \leq 4$, and, using Proposition 6 , it is easy to check that this system has the following integral solutions $\left(\nu_{2 a}, \nu_{4 a}, \nu_{8 a}, \nu_{8 b}\right)$ :

$$
\left\{\begin{array}{cccc}
(0,2,1,-2), & (0,2,-2,1), & (0,2,0,-1), & (0,2,-1,0), \\
(0,0,1,0), & (0,0,2,-1), & (0,0,0,1), & (0,0,-1,2)
\end{array}\right\} .
$$

In the case when $\left(\bar{\nu}_{2 a}, \bar{\nu}_{4 a}\right)=(2,-1)$, first using $\mathfrak{B C T}(11)$, by $(2.2)$ we obtain $\mu_{0}\left(u, \chi_{3}\right)=-\mu_{4}\left(u, \chi_{3}\right)=-\nu_{2 a}=0$ and

$$
\begin{aligned}
& \mu_{1}\left(u, \chi_{3}\right)=\frac{1}{8}\left(4 \nu_{8 a}-4 \nu_{8 b}+12\right) \geq 0 \\
& \mu_{5}\left(u, \chi_{3}\right)=\frac{1}{8}\left(-4 \nu_{8 a}+4 \nu_{8 b}+12\right) \geq 0 \\
& \mu_{0}\left(u, \chi_{2}\right)=\frac{1}{8}\left(4 \nu_{4 a}-4 \nu_{8 a}-4 \nu_{8 b}+12\right) \geq 0 \\
& \mu_{4}\left(u, \chi_{2}\right)=\frac{1}{8}\left(-4 \nu_{4 a}+4 \nu_{8 a}+4 \nu_{8 a}+12\right) \geq 0 \\
& \mu_{0}\left(u, \chi_{5}\right)=\frac{1}{8}\left(-4 \nu_{4 a}-4 \nu_{8 a}-4 \nu_{8 a}+28\right) \geq 0 \\
& \mu_{4}\left(u, \chi_{5}\right)=\frac{1}{8}\left(4 \nu_{4 a}+4 \nu_{8 a}+4 \nu_{8 a}+28\right) \geq 0
\end{aligned}
$$

It is easy to check that $-7 \leq \nu_{4 a} \leq 9,-4 \leq \nu_{8 a}, \nu_{8 b} \leq 4$, and the system has the following integral solutions $\left(\nu_{2 a}, \nu_{4 a}, \nu_{8 a}, \nu_{8 b}\right)$ :

$$
\begin{aligned}
& \{(0,2,-1,0), \quad(0,0,-1,2), \quad(0,2,0,-1), \quad(0,0,0,1) \text {, } \\
& (0,0,2,-1), \quad(0,2,-2,1), \quad(0,0,1,0), \quad(0,2,1,-2) \quad\} .
\end{aligned}
$$

It follows from (3.3)-(3.6) that the only four solutions which appear in both cases when $p=3$ and $p=11$ are the following ones: $\nu_{2 a}=0$ and

$$
\left(\nu_{4 a}, \nu_{8 a}, \nu_{8 b}\right) \in\{(0,0,1),(0,1,0),(2,-1,0),(2,0,-1)\} .
$$

Again, we were not able to produce a contradiction computing all possible $\mu_{i}\left(u, \chi_{j}\right)$ for all above listed tuples $\left(\nu_{4 a}, \nu_{8 a}, \nu_{8 b}\right)$ for the ordinary character table of $G$ as well as for $\mathfrak{B C T}(p)$, where $p \in\{3,5,11\}$. 
- Let $u$ be a unit of order 11 . Then using $\mathfrak{B C T}(3)$, by (2.2) we have

$$
\begin{aligned}
& \mu_{1}\left(u, \chi_{2}\right)=\frac{1}{11}\left(6 \nu_{11 a}-5 \nu_{11 b}+5\right) \geq 0 ; \\
& \mu_{2}\left(u, \chi_{2}\right)=\frac{1}{11}\left(-5 \nu_{11 a}+6 \nu_{11 b}+5\right) \geq 0,
\end{aligned}
$$

which has only the following trivial solutions $\left(\nu_{11 a}, \nu_{11 b}\right)=\{(1,0),(0,1)\}$.

For all the above mentioned cases except elements of orders 4, 6 and 8 we see that there is precisely one conjugacy class with non-zero partial augmentation. Thus, by Proposition 5, part (ii) of the Theorem is proved.

It remains to prove parts (i) and (vi), considering units of $V(\mathbb{Z} G)$ of orders 10 , $12,15,22,24,33$ and 55 . Now we treat each of these cases separately:

- Let $u$ be a unit of order 10. Then by (2.1), (3.1) and Proposition 4 we get $\nu_{2 a}+\nu_{5 a}=1$. Using $\mathfrak{B C T}(3)$, by (2.2) we have the system of inequalities

$$
\begin{aligned}
& \mu_{5}\left(u, \chi_{4}\right)=\frac{1}{10}\left(-8 \nu_{2 a}+8\right) \geq 0 \\
& \mu_{0}\left(u, \chi_{4}\right)=\frac{1}{10}\left(8 \nu_{2 a}+12\right) \geq 0 \\
& \mu_{2}\left(u, \chi_{2}\right)=\frac{1}{10}\left(-\nu_{2 a}+6\right) \geq 0
\end{aligned}
$$

which has no integral solutions such that $\mu_{5}\left(u, \chi_{4}\right), \mu_{0}\left(u, \chi_{4}\right), \mu_{2}\left(u, \chi_{2}\right) \in \mathbb{Z}$.

- Let $u$ be a unit of order 12. By (2.1), (3.1) and Proposition 4, we obtain that

$$
\nu_{2 a}+\nu_{3 a}+\nu_{4 a}+\nu_{6 a}=1 .
$$

Since $\left|u^{2}\right|=6$ and $\left|u^{3}\right|=4$, by (3.2) it yields that

$$
\chi_{j}\left(u^{2}\right)=\bar{\nu}_{2 a} \chi_{j}(2 a)+\bar{\nu}_{3 a} \chi_{j}(3 a)+\bar{\nu}_{6 a} \chi_{j}(6 a)
$$

and $\chi_{j}\left(u^{3}\right)=\widetilde{\nu}_{2 a} \chi_{j}(2 a)+\widetilde{\nu}_{4 a} \chi_{j}(4 a)$.

Consider the following four cases from parts (ii) and (iii) of the Theorem:

1. Let $\left(\bar{\nu}_{2 a}, \bar{\nu}_{3 a}, \bar{\nu}_{6 a}\right) \in\{(0,0,1),(2,-3,2),(-2,3,0)\}$ and suppose that

$$
\left(\widetilde{\nu}_{2 a}, \widetilde{\nu}_{4 a}\right) \in\{(0,1),(2,-1)\} .
$$

Then by $(2.2)$ we have $\mu_{0}\left(u, \chi_{2}\right)=\frac{1}{2} \notin \mathbb{Z}$, a contradiction.

2. Let $\left(\bar{\nu}_{2 a}, \bar{\nu}_{3 a}, \bar{\nu}_{6 a}\right)=(2,0,-1)$ and $\left(\widetilde{\nu}_{2 a}, \widetilde{\nu}_{4 a}\right) \in\{(0,1),(2,-1)\}$. Then by $(2.2)$ we have $\mu_{1}\left(u, \chi_{3}\right)=\frac{1}{2} \notin \mathbb{Z}$, a contradiction.

3. Let $\left(\bar{\nu}_{2 a}, \bar{\nu}_{3 a}, \bar{\nu}_{6 a}\right)=(0,3,-2)$ and $\left(\widetilde{\nu}_{2 a}, \widetilde{\nu}_{4 a}\right)=(0,1)$. According to (2.2), $\mu_{6}\left(u, \chi_{6}\right)=-\mu_{0}\left(u, \chi_{6}\right)=\frac{2}{3} \nu_{3 a}=0$, so $\nu_{3 a}=0$, and we have the system

$$
\begin{aligned}
& \mu_{2}\left(u, \chi_{5}\right)=\frac{1}{12}\left(6 \nu_{2 a}-2 \nu_{4 a}+8\right) \geq 0 \\
& \mu_{4}\left(u, \chi_{5}\right)=\frac{1}{12}\left(-6 \nu_{2 a}+2 \nu_{4 a}+4\right) \geq 0 \\
& \mu_{2}\left(u, \chi_{3}\right)=\frac{1}{12}\left(-4 \nu_{2 a}+2 \nu_{6 a}+6\right) \geq 0 \\
& \mu_{4}\left(u, \chi_{3}\right)=\frac{1}{12}\left(4 \nu_{2 a}-2 \nu_{6 a}+6\right) \geq 0 \\
& \mu_{4}\left(u, \chi_{2}\right)=\frac{1}{12}\left(-4 \nu_{2 a}-4 \nu_{4 a}+2 \nu_{6 a}+10\right) \geq 0 \\
& \mu_{2}\left(u, \chi_{2}\right)=\frac{1}{12}\left(4 \nu_{2 a}+4 \nu_{4 a}-2 \nu_{6 a}+2\right) \geq 0
\end{aligned}
$$

that has only two solutions $\{(-1,0,1,1),(1,0,1,-1)\}$ with $\mu_{i}\left(u, \chi_{j}\right) \in$ $\mathbb{Z}$. 
4. Let $\left(\bar{\nu}_{2 a}, \bar{\nu}_{3 a}, \bar{\nu}_{6 a}\right)=(0,3,-2)$ and $\left(\widetilde{\nu}_{2 a}, \widetilde{\nu}_{4 a}\right)=(2,-1)$. Using $(2.2)$, we get $\mu_{6}\left(u, \chi_{6}\right)=-\mu_{0}\left(u, \chi_{6}\right)=\frac{2}{3} \nu_{3 a}=0$, so $\nu_{3 a}=0$. Put $t=2 \nu_{2 a}-\nu_{6 a}$. Then by (2.2)

$$
\mu_{0}\left(u, \chi_{3}\right)=\frac{1}{12}(-4 t+4) \geq 0 ; \quad \mu_{4}\left(u, \chi_{3}\right)=\frac{1}{12}(2 t-2) \geq 0,
$$

so $2 \nu_{2 a}-\nu_{6 a}=1$. Now by $(2.2)$ we have

$$
\begin{aligned}
& \mu_{2}\left(u, \chi_{5}\right)=\frac{1}{12}\left(2\left(3 \nu_{2 a}-\nu_{4 a}\right)-8\right) \geq 0, \\
& \mu_{0}\left(u, \chi_{9}\right)=\frac{1}{12}\left(-4\left(3 \nu_{2 a}-\nu_{4 a}\right)+28\right) \geq 0,
\end{aligned}
$$

and $3 \nu_{2 a}-\nu_{4 a}=4$. Using (2.1) we obtain that $\nu_{2 a}=\nu_{4 a}=-\nu_{6 a}=1$. Finally, $\mu_{4}\left(u, \chi_{9}\right)=\frac{1}{12}\left(6 \nu_{2 a}-2 \nu_{4 a}+28\right)=\frac{8}{3} \notin \mathbb{Z}$, a contradiction. Thus, part (vi) of the Theorem is proved.

- Let $u$ be a unit of order 15 . Then by (2.1) and (3.1) we have $\nu_{3 a}+\nu_{5 a}=1$. Now using the character table of $G$, by (2.2) we get the system of inequalities

$$
\mu_{0}\left(u, \chi_{2}\right)=\frac{1}{15}\left(8 \nu_{3 a}+12\right) \geq 0 ; \quad \mu_{5}\left(u, \chi_{2}\right)=\frac{1}{15}\left(-4 \nu_{3 a}+9\right) \geq 0,
$$

that has no integral solutions such that $\mu_{0}\left(u, \chi_{2}\right), \mu_{5}\left(u, \chi_{2}\right) \in \mathbb{Z}$.

- Let $u$ be a unit of order 22. Then by (2.1), (3.1) and Proposition 4 we obtain that

$$
\nu_{2 a}+\nu_{11 a}+\nu_{11 b}=1 \text {. }
$$

In (2.2) we need to consider two cases: $\chi\left(u^{2}\right)=\chi(11 a)$ and $\chi\left(u^{2}\right)=\chi(11 b)$, but in both cases by $(2.2)$ we have

$$
\mu_{0}\left(u, \chi_{2}\right)=-\mu_{11}\left(u, \chi_{2}\right)=\frac{1}{22}\left(20 \nu_{2 a}-10 \nu_{11 a}-10 \nu_{11 b}+2\right)=0 .
$$

It yields $10 \nu_{2 a}-5 \nu_{11 a}-5 \nu_{11 b}=-1$, that has no integral solutions.

- Let $u$ be a unit of order 24. Then by (2.1) and (3.1) we have

$$
\nu_{2 a}+\nu_{3 a}+\nu_{4 a}+\nu_{6 a}+\nu_{8 a}+\nu_{8 b}=1 .
$$

Since $\left|u^{2}\right|=12,\left|u^{4}\right|=6,\left|u^{3}\right|=8,\left|u^{6}\right|=4$, and $G$ has two conjugacy classes of elements of order 8 , we need to consider 40 various cases defined by parts (iii)-(vi) of the Theorem.

Let $\left(\bar{\nu}_{2 a}, \bar{\nu}_{3 a}, \bar{\nu}_{6 a}\right) \in\{(0,3,-2),(2,0,-1)\}$, where

$$
\chi_{j}\left(u^{4}\right)=\bar{\nu}_{2 a} \chi_{j}(2 a)+\bar{\nu}_{3 a} \chi_{j}(3 a)+\bar{\nu}_{6 a} \chi_{j}(6 a) .
$$

According to $(2.2)$ we have that $\mu_{1}\left(u, \chi_{2}\right)=\frac{1}{2} \notin \mathbb{Z}$, a contradiction. In the remaining cases similarly we obtain that $\mu_{1}\left(u, \chi_{2}\right)=\frac{1}{4} \notin \mathbb{Z}$, which is also a contradiction.

- Let $u$ be a unit of order 33. Then by (2.1) and (3.1) we have $\nu_{3 a}+\nu_{11 a}+$ $\nu_{11 b}=1$. Again, in $(2.2)$ we need to consider two cases: $\chi\left(u^{3}\right)=\chi(11 a)$ and $\chi\left(u^{3}\right)=\chi(11 b)$, but both cases lead us to the same system of inequalities

$$
\mu_{1}\left(u, \chi_{5}\right)=\frac{1}{33}\left(2 \nu_{3 a}+9\right) \geq 0 ; \quad \mu_{11}\left(u, \chi_{5}\right)=\frac{1}{33}\left(-20 \nu_{3 a}+9\right) \geq 0,
$$

which has no integral solutions with $\mu_{1}\left(u, \chi_{5}\right), \mu_{11}\left(u, \chi_{5}\right) \in \mathbb{Z}$. 
- Let $u$ be a unit of order 55. Then by (2.1) and (3.1) we have $\nu_{5 a}+\nu_{11 a}+\nu_{11 b}=$ 1. Considering two cases when $\chi\left(u^{5}\right)=\chi(11 a)$ and $\chi\left(u^{5}\right)=\chi(11 b)$, we get the same systems of inequalities

$$
\mu_{5}\left(u, \chi_{8}\right)=\frac{1}{55}\left(4 \nu_{5 a}+40\right) \geq 0 ; \quad \mu_{5}\left(u, \chi_{5}\right)=\frac{1}{55}\left(-4 \nu_{5 a}+15\right) \geq 0,
$$

which also has no integral solutions such that $\mu_{5}\left(u, \chi_{5}\right), \mu_{5}\left(u, \chi_{8}\right) \in \mathbb{Z}$.

Thus, the theorem is proved, and now the corollary follows immediately.

\section{References}

[1] V. A. Artamonov and A. A. Bovdi, Integral group rings: groups of invertible elements and classical K-theory, in Algebra. Topology. Geometry, Vol. 27 (Russian), 3-43, 232, Akad. Nauk SSSR, Vsesoyuz. Inst. Nauchn. i Tekhn. Inform., Moscow. MR1039822 (91e:16028). Translated in J. Soviet Math. 57 (1991), no. 2, 2931-2958.

[2] S. D. Berman, On the equation $x^{m}=1$ in an integral group ring, Ukrain. Mat. Ž. 7 (1955), 253-261. MR0077521 (17,1048g)

[3] F. M. Bleher and W. Kimmerle, On the structure of integral group rings of sporadic groups, LMS J. Comput. Math. 3 (2000), 274-306 (electronic). MR1783414 (2001i:20006)

[4] V. Bovdi, C. Höfert and W. Kimmerle, On the first Zassenhaus conjecture for integral group rings, Publ. Math. Debrecen 65 (2004), no. 3-4, 291-303. MR2107948 (2006f:20009)

[5] V. Bovdi, A. Konovalov, R. Rossmanith and C. Schneider, LAGUNA - Lie AlGebras and UNits of group Algebras, v.3.3.3; 2006 (http://ukrgap.exponenta.ru/laguna.htm).

[6] J. A. Cohn and D. Livingstone, On the structure of group algebras, I, Canad. J. Math. 17 (1965), 583-593. MR0179266 (31 \#3514)

[7] The GAP Group, GAP - Groups, Algorithms, and Programming, Version 4.4; 2006 (http://www.gap-system.org).

[8] D. Gorenstein, The classification of finite simple groups, Vol. 1, Plenum, New York, 1983. MR0746470 (86i:20024)

[9] M. Hertweck, Partial augmentations and Brauer character values of torsion units in group rings, to appear (2005), 26 pages.

[10] M. Hertweck, On the torsion units of some integral group rings, Algebra Colloq. 13 (2006), no. 2, 329-348. MR2208368

[11] W. Kimmerle, On the prime graph of the unit group of integral group rings of finite groups, in Groups, rings and algebras, Contemporary Mathematics, AMS, to appear.

[12] I. S. Luthar and I. B. S. Passi, Zassenhaus conjecture for $A_{5}$, Proc. Indian Acad. Sci. Math. Sci. 99 (1989), no. 1, 1-5. MR1004634 (90g:20007)

[13] I. S. Luthar and P. Trama, Zassenhaus conjecture for $S_{5}$, Comm. Algebra 19 (1991), no. 8, 2353-2362. MR1123128 (92g:20003)

[14] Z. Marciniak, J. Ritter, S. K. Sehgal and A. Weiss, Torsion units in integral group rings of some metabelian groups, II, J. Number Theory 25 (1987), no. 3, 340-352. MR0880467 (88k:20019)

[15] H. Zassenhaus, On the torsion units of finite group rings, in Studies in mathematics (in honor of A. Almeida Costa) (Portuguese), 119-126, Inst. Alta Cultura, Lisbon. MR0376747 (51 \#12922) 